\title{
SEVERE EVOLUTION WITH MULTIPLE SEPTIC DETERMINATIONS IN A PATIENT DIAGNOSED WITH STREPTOCOCCUS GALLOLYTICUS INFECTION
}

\author{
Constantin Marilena ${ }^{1}$, Neața Mădălina ${ }^{1}$, Ștefan Liliana ${ }^{1}$, Acatrinei Eliza ${ }^{1}$, \\ Bumbu Aurelia $^{2}$, Enyedi Mihai ${ }^{1}$, Nica Maria ${ }^{1}$, Voinea Cristina ${ }^{1}$, Calistru Petre ${ }^{1}$ \\ 1 "Dr. Victor Babes" Foundation \\ 2 "Dr. Victor Babes" Hospital
}

\begin{abstract}
Streptococcus gallolyticus, member of streptococcus bovis group colonizes digestive tubes of birds, cows and human $(2.5-15 \%)$. Literature describes the association between bacteriemia with Streptococcus gallolyticus and colon tumours but also extracolonic lesions (bacterial endocarditis, vertebral osteomyelitis, dyscitis, colangitis, lung tumors, ovarian tumors).

We report the case of a 60 years old male caucasian patient who was diagnosed with vertebral osteomyelitis as the first lesion of Streptococcus gallolyticus infection identified by blood culture. Other lesions were diagnosed on aortic valve (bacterian endocarditis), right popliteal artery (popliteal artery thrombosis), right knee (arthritis). Even if there was a clinical and biological improvement during the treatment, the imaging tests done after 30 and 45 days from the diagnosis showed severe aortic insuficiency so surgical intervention for aortic valvuloplasty was done in a short time. Vertebral and popliteal artery lesions have improved within two months of antibiotic treatment. Colonoscopy, which was done 6 weeks from the diagnosis showed colonic polyps - risk factors of Streptococcus gallolyticus bacteriemia.

This case proves that Streptococcus gallolyticus infection has a silent period followed by severe evolution by multiple septic lesions inspite of the precocious treatment and warn of the necessity of digestive investigations at these patients.
\end{abstract}

Keywords: Streptococcus Gallolyticus infection, vertebral osteomyelitis, endocarditis, thromboses

\section{INTRODUCTION}

Streptococcus Gallolyticus, a member of Streptococcus Bovis group is an opportunistic microorganism that colonizes digestive tube of birds, cows and human, but in the presence of predisposing factors (incipient colonic lesions) may induce bacteriemia with multiple septic determinations. Streptococcus Gallolitycus infection is associated with gastrointestinal neoplasia (polyps and colon carcinoma) usually diagnosed after an episode of endocarditis and responds to Penicillin treatment (5).
Data from literature shows that 25-80\% of patients with cu S.Bovis have colorectal tumours, $18-62 \%$ of the patients have bacterial endocarditis and colon neoplasia, 94\% of S.Bovis bacteriemia are due to type 1 (Streptococcus Gallolyticus) and are associated with colorectal tumors (1).

In 1951 Mc Coy and Mason suggested the relationship between colon carcinoma and bacterial endocarditis but in 1974 the association between S.Bovis and colorectal cancer was recognized (1).

Corresponding author:

Constantin Marilena, “Dr. Victor Babes Foundation”, 281 Mihai Bravu Avenue, District 3, Bucharest

E-mail: marilena.constantin@cdt-babes.ro 
The most frequent extracolonic manifestations are: osteomyelitis, diskitis, neck abscess (1).

Possible mechanisms for Streptococcus Gallolyticus to induce colon cancer and bacterial endocarditis are described in literature: chronic inflammation, promotion of angiogenesis, increasing vascular permeability, uncontrolled cellular proliferation (1). Streptococcus Gallolyticus has two essential factors of virulence represented by polysaccharides capsules and pili structures which are implicated in paracellular translocations, capacity to escape innate immune system, resistance to complement system. Streptococcus Gallolyticus remains in circulation and adheres to endocardium with biofilm formation (2).

\section{CASE PRESENTATION}

A 60 years old, male, caucasian patient presented to my office with very intense right back pain in July 2015. He accused fatigue and had lost about $6 \mathrm{~kg}$ in the last 3-4 months. In June 2015 he had anitis and urinary infection with E. Coli treated with Cefuroxime, but also an episode of diplopia. Twenty years ago he was operated for left knee menisc fracture. The patient is a smoker (10-15 cigarettes per day) and drinks alcohol occasionally. His medical family history is relevant for: colon cancer (mother), pulmonary tumor (father), leukemia (sister).

Clinical exam showed an overweight patient $(\mathrm{BMI}=28.7 \mathrm{~kg} / \mathrm{m} 2)$ with medium physical condition, normal pulmonary exam, HR=64/min, $\mathrm{BP}=100 / 70 \mathrm{mmHg}$, right flank pain and right lumbar pain.

Blood tests described $\mathrm{WBC}=9170 / \mathrm{mm} 3$, neutrophilia (87.7\%), inflammatory syndrome (ESR $=51 \mathrm{~mm} / \mathrm{h}, \mathrm{Fib}=552 \mathrm{mg} / \mathrm{dl}, \mathrm{CRP}=8.3 \mathrm{mg} / \mathrm{dl}$, total protein $=7.2 \mathrm{~g} / \mathrm{dl}$, albumine $=55.9 \%$, alfa1globuline $=4.3 \%$, iron $=65 \mathrm{mcg} / \mathrm{dl}$, serum ferritine $=903 \mathrm{mcg} / \mathrm{dl}$ ), positive rheumatic factor, enlarged spleen (abdominal ultrasound).t

Lumbar MRI showed disk hernia with anterolistesis L5-S1. Cerebral MRI described unspecific demyelinating lesions.
Clinical exam and investigations guided us to the diagnosis of: disk hernia; inflammatory syndrome. We excluded other causes for inflammatory syndrome (rheumatic, hematological, pulmonary, prostate causes).

Under the treatment with NSAI drugs, dexametasone and tramadolum the patient continued to have right lumbar pain and he began to present fever. He was hospitalized on $10^{\text {th }}$ of August.

Blood tests showed leucocytosis (16200/ $\mathrm{mm} 3)$, neutrophilia (86\%), lymfopenia (7.4\%), thrombocytopenia (141000/mm3), inflammatory syndrome $(E S R=37 \mathrm{~mm} / \mathrm{h}$, fibrinogen = $488 \mathrm{mg} / \mathrm{dl}$, CRP = $3.3 \mathrm{mg} / \mathrm{dl}$ ). Urine exam was normal.

Ecocardiography done in the first day of admission and repeated two days later described mild aortic insufficiency but no vegetations.

Blood culture was done.

We initiated intravenous treatment with Amoxipenicillin $2.4 \mathrm{~g} /$ day and Gentamicine 160 $\mathrm{mg} /$ day.

Table 1. Blood culture was positive for Streptococcus gallolyticus in the forth day of hospitalization. Susceptibility test to antibiotic was done (VITEK system)

\begin{tabular}{|l|c|l|}
\hline & CMI & interpretation \\
\hline Benzylpenicili & $\leq 0.6$ & $\mathrm{~S}$ \\
\hline Ampicillin & $\leq 0.25$ & $\mathrm{~S}$ \\
\hline Cefotaxim & $\leq 0.12$ & $\mathrm{~S}$ \\
\hline Ceftriaxon & $\leq 0.12$ & $\mathrm{~S}$ \\
\hline Levofloxacin & 4 & $\mathrm{I}$ \\
\hline Erithromycin & $\leq 0.12$ & $\mathrm{~S}$ \\
\hline Clindamycin & $\leq 0.25$ & $\mathrm{~S}$ \\
\hline Linezolid & $\leq 2$ & $\mathrm{~S}$ \\
\hline Vancomycin & 0.25 & $\mathrm{~S}$ \\
\hline Tetracycline & $\geq 0.16$ & $\mathrm{R}$ \\
\hline
\end{tabular}

Persistence of right lumbar pain and fever imposed repeating lumbar MRI which described inflamatory lesion in vertebra L1 (Fig. 1)

Two days after hospitalizing patient described pain in the left lower limb in standing position with decreasing intensity in sitting position. Ddimeri test was positive (3589 ng/ml), Doppler ultrasound for veins and arteries and lower limb angio-CT established the diagnosis of left popliteal artery thrombosis (Fig. 2). 


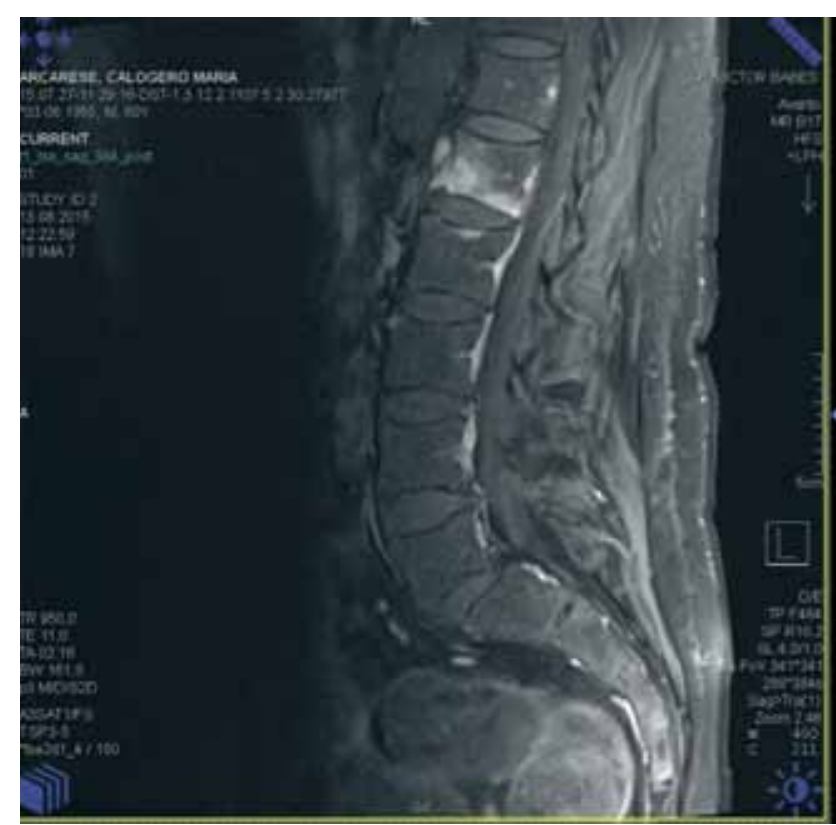

Figure 1. Lumbar RMI describes inflamatiory lesions in L1

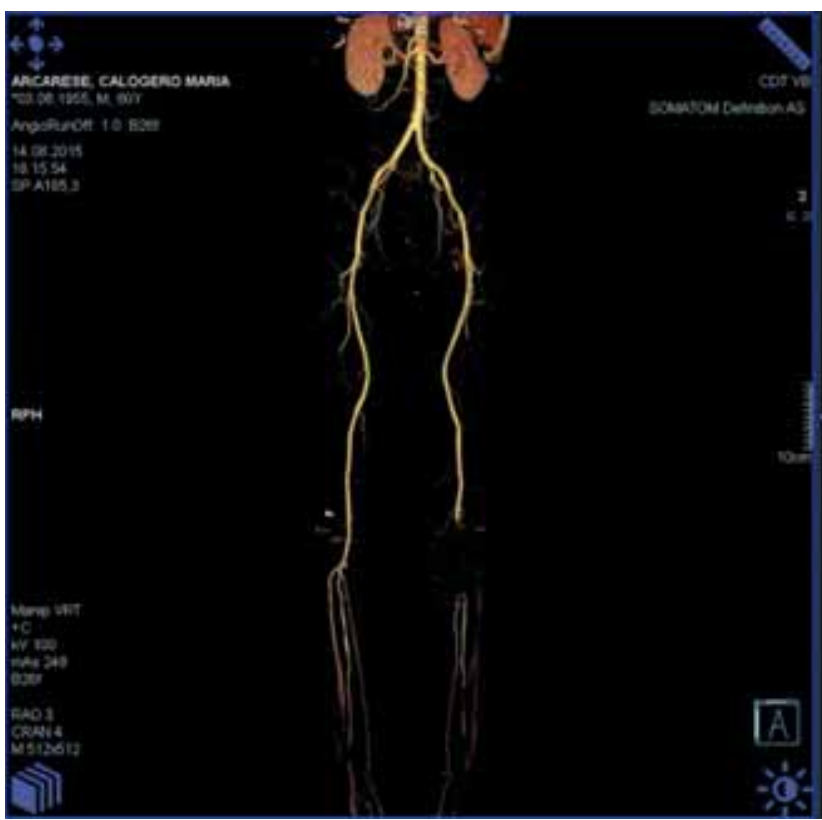

Figure 2. Angio CT for lower limb describes the absence of blood stream in the terminal level of left popliteeal artery, the emergencies and proximal levels of anterior and posterior tibial arterieris and fibular artery up to the linkage between the upper third with the lower two thirds of gluteal region

We initiated anticoagulant therapy with enoxaparine in therapeutical dose. Vascular surgery consultation recommended anticoagulant therapy with heparin, and arteriography. It was not recommended emergency surgical intervention for artery thrombosis.

Pulmonary embolism was excluded by chest tomography with contrast substance.
In the sixth day of hospitalization we repeated transthoracic ecocardiogram which described possible vegetations of 9 and $4 \mathrm{~mm}$ on the aortic valve.We started the bacterial endocarditis protocol with Penicillin G 18 millions/ day (in six doses) and Gentamicine $240 \mathrm{mg} /$ day (in three doses).

Two days later transesofageal echocardiogram confirmed the diagnosis of endocarditis, but it was a lot of uncertainty about the sterility of vegetations. Taking in consideration clinical context we decided to continue the protocol for infectious endocarditis described above. There was no indications for surgery intervention at that moment.

After ten days of treatment we tried to decrease Penicillin dose to 12 millions/day but fever reappeared with an episode of left knee arthritis.

Table 2. Positive diagnosis

\begin{tabular}{|l|l|}
\hline Sepsis & $\begin{array}{l}4 \text { criteria } \\
\text { fever }>38 \text { grd. C; tachycardia > 90/min; } \\
\text { leucocytosis > 12000/mm3; positive blood } \\
\text { culture }\end{array}$ \\
\hline $\begin{array}{l}\text { Infectious } \\
\text { endocarditis with } \\
\text { Streptococcus } \\
\text { Gallolyticus }\end{array}$ & $\begin{array}{l}\text { Duke criteria: } \\
\text { one major criteria: ecocardiography } \\
\text { 4 minor criteria: fever } \geq 38 \text { grd.C, vascular } \\
\text { phenomena (major arterial embolism), } \\
\text { immunological phenomena (positive } \\
\text { rheumatoid factor), microbiological criteria } \\
\text { (positive blood culture) without meet a } \\
\text { major criteria }\end{array}$ \\
\hline $\begin{array}{l}\text { Vertebral } \\
\text { osteomyelitis }\end{array}$ & $\begin{array}{l}\text { fever; lumbar pain; inflamatory syndrome; } \\
\text { positive blood culture; lumbar RMl; } \\
\text { predisposing factors (degenerative lesions } \\
\text { of lumbar column) }\end{array}$ \\
\hline $\begin{array}{l}\text { Popliteal artery } \\
\text { thrombosis }\end{array}$ & $\begin{array}{l}\text { Positive D-dimeri; Doppler ultrasound of } \\
\text { lower limb artery; angio-CT }\end{array}$ \\
\hline Left knee arthritis & $\begin{array}{l}\text { Clinical exam: tumefaction, inflammation, } \\
\text { pain }\end{array}$ \\
\hline Disk hernia L5-S1 & Lumbar MRI \\
\hline
\end{tabular}

Patient was followed up during hospitalization by monitorig temperature every four hours, blood presure and heart rate. We did periodic evaluation by ECG, transthoracic ecocardiography, Doppler ultrasound for lower limb arteries. We used CRP to monitor inflammatory syndrome. We made the patient recommendations of minimal mobilization, wearing thoraco-lumbar corset, passive mobilization of lower limbs, hydration. 
Table 3. Antibiotic treatment during hospitalization

Week 33

Week 41

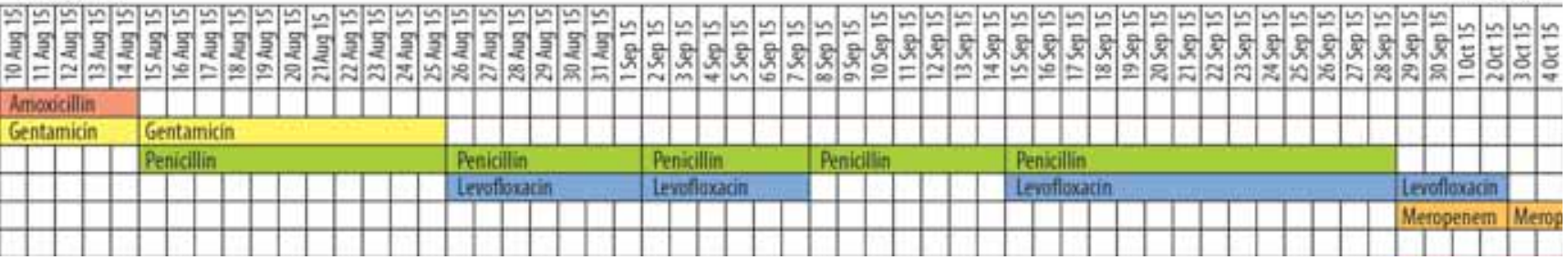

Regarding to antibiotic treatment we respected the standard protocol for infective endocarditis but taking in consideration vertebral lesions we cotinued the treatment with fluoroquinolones and aminopenicilline up to six months. Associated treatment consisted of: enoxaparine, acetylsalicylic acid, pantoprazole, gabapentin, probiotic (Table 3 ).

Under the treatment the evolution of the patient was favorable both from clinical and biological point of view(no fever, improved lumbar pain, no pain on the left lower limb, improved inflamatory phenomena on the left knee, improved inflamatory syndrome), but repeated transthoracic and transesofageal echocardiography on

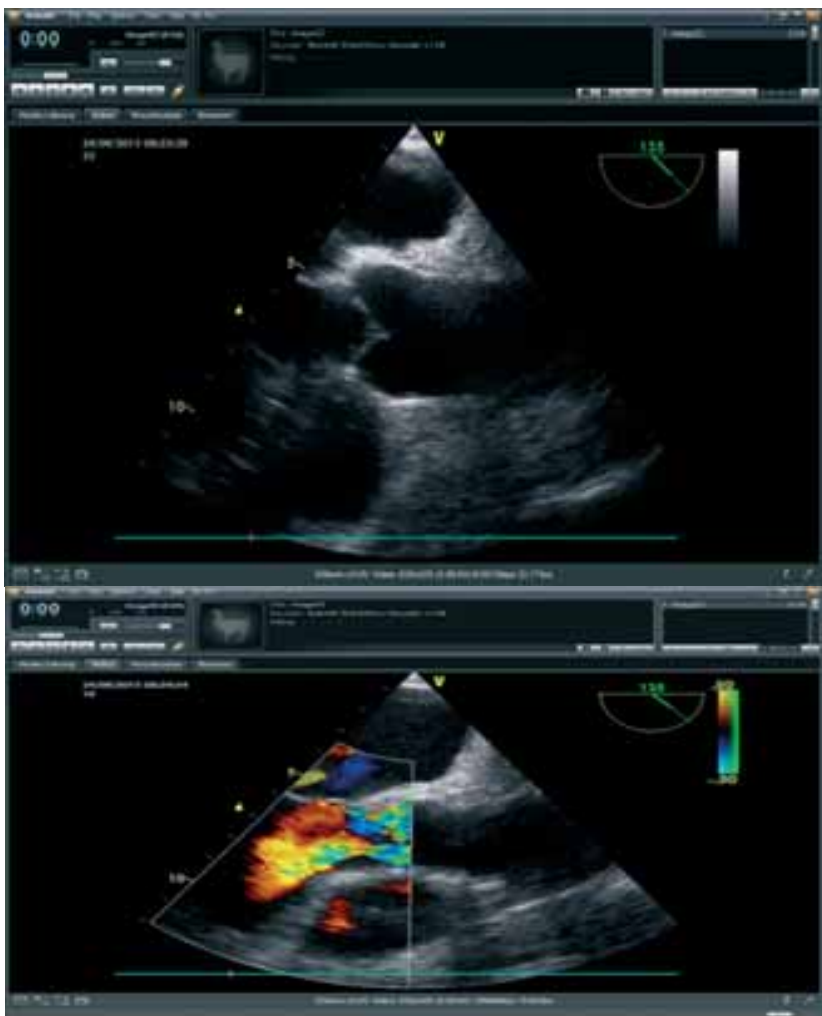

Figure 3. Transesofagieal ecocardiography describes severe aortic insuficiency and mobile lesions sugestive for vegetations; medium dilatation of the left atrium; dilatation of left ventricule $61 / 44 \mathrm{~mm}$ day forty-five of hospitalization and after fourty days of treatment with Penicilline described severe aortic insuficiency with indication for valve replacement (Fig. 3).

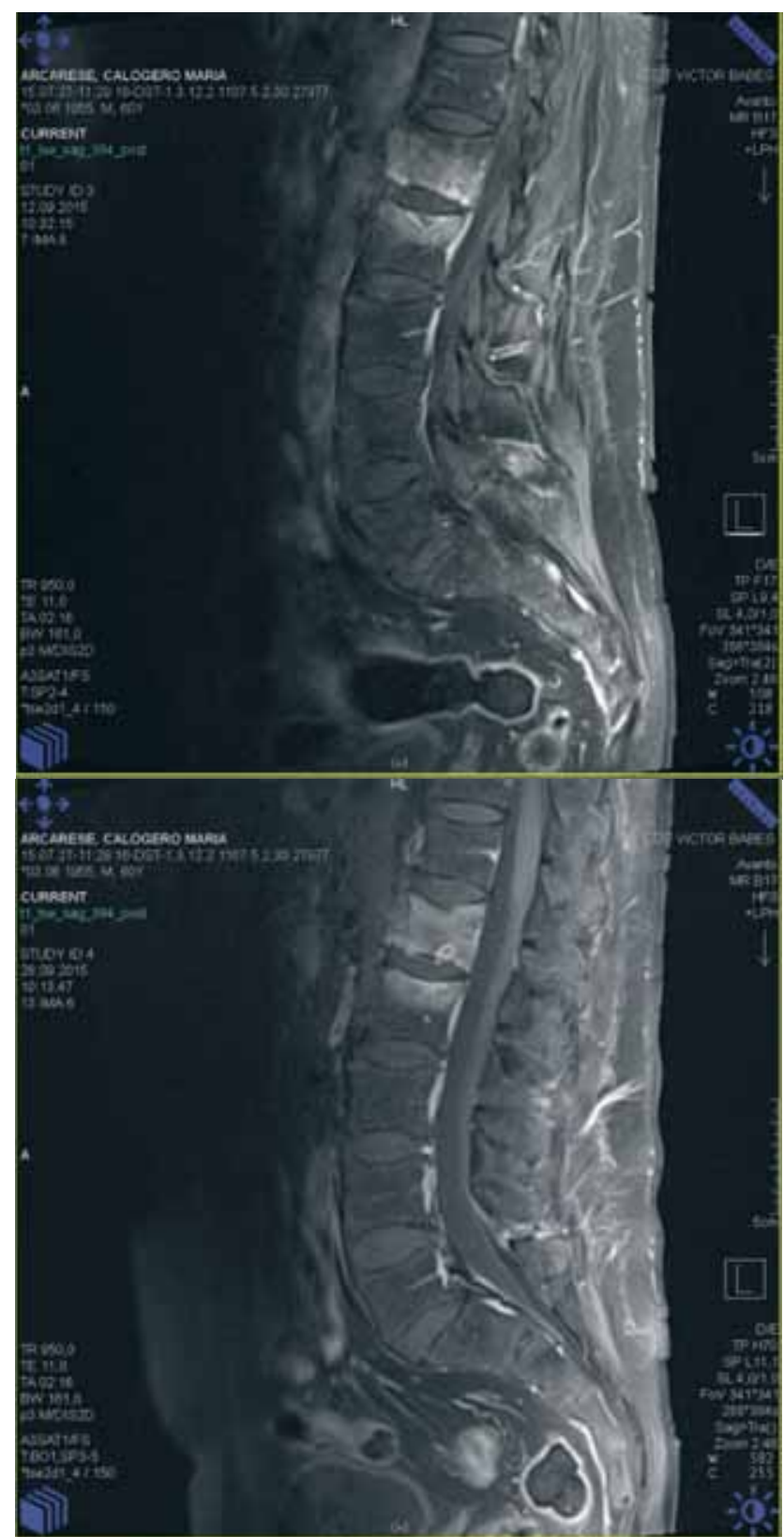

Figure 4. Lumbar column MRI described inflammatory lesions at T12-L2 level in progression comparative to the last exam and degenerative vertebral lesions 
Lumbar MRI done after thirty-two days of hospitalization showed vertebral lesions in progression comparative the last exam (Fig. 4).

Doppler Ultrasound for left popliteeal artery was repeated on day forty-five of hospitalization with no changes of thrombus image.

On Octomber 2015 patient was admitted to Niguarda Hospital - Milano where surgical intervention for aortic valve replacement with biological valve was done(vegetations were sterile at the moment of surgical intervention) with favorable evolution after surgery.

Colonoscopy performed at Niguarda Hospital on Octomber identified two colonic polyps and polypectomy was done (histopatological exame on working).

Doppler ultrasound for the left polpiteal artery repeated at Niguarda Hospital showed partial repermeabilisation of left popliteal artery.

The last lumbar MRI which was done three months and two weeks after begining antibiotic treatment (on november 2015) described an improvement of lesions (Fig. 5).

\section{DISCUSSION}

This case represented a diagnosis and treatment challenge. We could explain lumbar pain

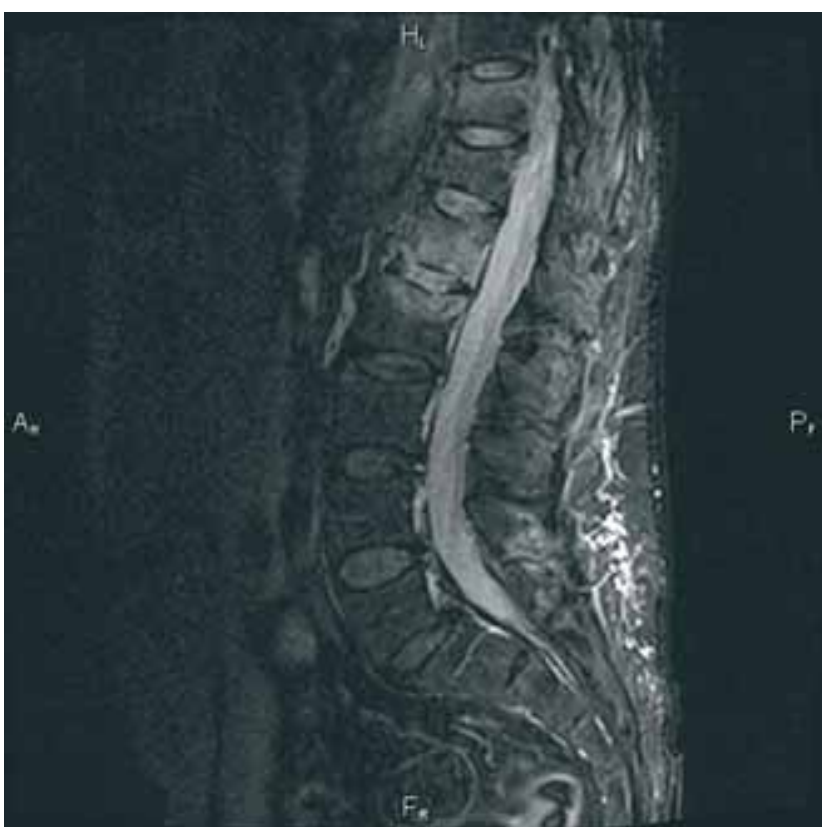

Figure 5. LT12-L2 lesions compatible with osteodiscitis in mild improvement; unchanged T11 lesions by MRI changes but the presence of inflammatory syndrome, fatigue, weight loss imposed other investigations that showed positive rheumatoid factor and splenomegaly. Fever was a key element for diagnosis, the association fever- lumbar pain - inflammatory syndrome imposed repeating lumbar MRI and established the diagnosis of vertebral osteomyelitis. Literature describes the association between osteomyelitis and bacterial endocarditis. The second key element in this case was identification of Streptococcus Gallolyticus in blood culture. Its association with bacterial endocarditis is well known. Studying literature data induced the initiation of treatment protocol of infectious endocarditis before the confirmation of diagnosis by transesofageal ecocardiography. Popliteal artery thrombosis and left knee arthritis were septic determinations of bacterial endocarditis. Antibiotic therapy was chosen taking in consideration the antibiotic susceptibility of Streptococcus Gallolyticus to antibiotics, treatment protocols for bacterial endocarditis and the penetrance of antibiotic into the bone.

Another question in this case was how Streptococcus Gallolyticus induced bacteriemia. Literature data $(1,2)$ (describe the association between Streptococcus Gallolyticus bacteriemia and colon tumours. Even if colonoscopy was postponed because of the clinical condition of the patient, it was done two months later from the diagnosis and confirmed the presence of colonic polyps- possible entrance gate for Streptococcus Gallolyticus. It is worth to be mentioned that septic determinations appeared in damaged zones (lumbar column with ostheophitosis, left knee affected by surgical intervention for broken meniscus).

Studying literature data shows that relationship Streptococcus Gallolyticus-colorectal tumours-bacterial endocarditis has a central element - collagen type IV- which is presented at the level of basal membrane of colonic mucosa and endocard (Streptococcus Gallolyticus colonized $10 \%$ of normal population, but $55 \%$ of 
patients with colonic lesions). Bacteria adhere by pili structures and form biofilm (2).

The particularity of the case results from difficulty to establish diagnosis but also to elaborate therapeutic management in the context of multiple septic determinations (endocarditis, osteomyelitis, arthritis, arterial thrombosis) that followed a silent evolution (fatigue, low

\section{REFERENCES}

1. Ahmed S. Abdulamir, Rand R. Hafidh, Fatimah Abu Bakar. The association of Streptococcus bovis/gallolyticus with colorectal tumors: The nature and the underlying mechanisms of its etiological role. Journal of Experimental \& Clinical Cancer Research. 2011; 30:11

2. Bolei A. Boleij, Muytjens C., Bukhari S., Cayet N. Novel clues on the specific association of Streptococcus gallolyticus subsp gallolyticus with colorectal cancer. The Journal of Infectious Disease. 2011, 203:1101-9 grade fever, weight loss). Extracolonic manifestations preceded the identification of colonic lesions (colonoscopy which was done later identified colonic polyps-possible entrance gate for etiological agent).

This case shows the gravity of Streptococcus Gallolyticus infection and draws attention to investigate digestive system in these patients.
3. Kowalski T.J., Layton K.F., Berbari E.F. Follow-up MR imaging in patients with Pyogenic spine Infection: Lack of Correlation with Clinical Features. AJNRAmJ Nueroradiology. 2007, 28:693-9

4. Zimmerli W. Vertebral Osteomyelitis. NEJM. 2010; 362: 1022-1029

5. Longo D., Kasper D., Jameson L., Fauci A., Hauser S., Loacalzo J. Harrison's Priciples of Internal Medicine. $18^{\text {th }}$ Edition, 2012

Tice A. Osteomyelitis; Wessels R. M. Streptococcal infection; Karchmer A.W. Infective Endocarditis 